\title{
Barrett-Ösophagus
}

\section{Overkill für einen Killer?}

\begin{abstract}
Der Barrett-Ösophagus ist eine Präkanzerose für das distale Ösophaguskarzinom. Die Häufigkeit dieses Tumors hat in den letzten Jahren deutlich zugenommen. Doch wie sollten Sie einen Patienten mit einem Barrett-Ösophagus behandeln? Ist wirklich eine Dauertherapie mit einem Protonenpumpenhemmer nötig und wann sollte die Barrett-Schleimhaut abgetragen werden?
\end{abstract}

- Von einem Barrett-Ösophagus spricht man, wenn das normale Plattenepithel der Speiseröhre durch eine intestinale Metaplasie ersetzt wird. Ursache dieser metaplastischen Veränderung ist eine chronische Refluxkrankheit. Die Bedeutung des BarrettÖsophagus als Präkanzerose gilt heute als gesichert. Man nimmt an, dass bei Patienten mit Barrett-Ösophagus das Risiko, ein Ösophaguskarzinom zu entwickeln, 0,5\% pro Jahr beträgt. Es ist damit erheblich höher als bei der allgemeinen Bevölkerung, so Prof. Prateek Sharma, Kansas City. Das absolute Risiko, ein Ösophaguskarzinom zu bekommen, ist allerdings auch bei Patienten mit einem Barrett-Ösophagus sehr niedrig.

\section{Wann ist Screening sinnvoll?}

In den vergangenen Jahren ist die Inzidenz des Adenokarzinoms der Speiseröhre stark angestiegen. Angesichts dieser epidemiologischen Entwicklung stellt sich die Frage, bei welchen Patienten mit chronischem Reflux ein Screening auf Barrett-Ösophagus durchgeführt werden sollte. Darauf gibt es bisher keine endgültige Antwort, so Sharma. Als Risikofaktoren für einen BarrettÖsophagus gelten weiße Hautfarbe, männliches Geschlecht, mittleres Alter, Patienten, bei denen in jungen Jahren bereits Refluxsymptome aufgetreten sind, und Patienten mit lang andauernder Refluxsymptomatik (über fünf Jahre).

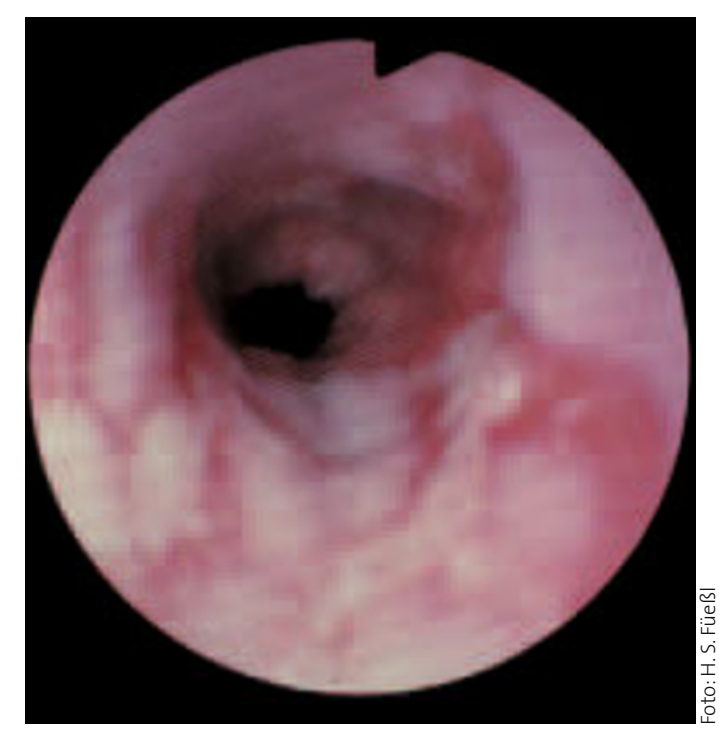

beantworten. In Studien konnte allerdings gezeigt werden, dass ein anhaltender Säurereflux für eine weitere Proliferation der Barrett-Schleimhaut prädisponiert. Bisher konnte jedoch nicht eindeutig belegt werden, dass eine Säuresuppressionstherapie zu einer Senkung des Karzinomsrisikos führt, auch wenn es unter der Protonenpumpenhemmertherapie zu einer Abheilung des Barrett-Ösophagus gekommen ist. Gleiches gilt für die chirurgische Therapie. Auch durch eine Fundoplicatio kann das Adenokarzinomrisiko nicht gesenkt werden.

\section{Wann ist die Mukosektomie indiziert?}

Auch die endoskopische Behandlung eines Barrett-Ösophagus, die endoskopische Mukosektomie, bietet keinen vollständigen Schutz vor einem Karzinom. Selbst wenn nach einem solchen Eingriff die Barrett-Schleimhaut durch neues Plattenepithel ersetzt wird, findet sich nicht selten noch metaplastisches Gewebe unter dem neuen Plattenepithel, das Ausgangspunkt für ein Karzinom sein kann. Deshalb empfiehlt sich bei Barrett-Ösophagus-Patienten ohne oder mit nur geringen Dysplasien die endoskopische Abtragung der Barrett-Schleimhaut nicht. Dagegen ist bei Patienten mit hochgradiger Dysplasie oder intramukösem Karzinom die endoskopische Entfernung heute eine sehr sinnvolle und erfolgreiche kurative Behandlungsoption. Eine Alternative ist die fotodynamische Therapie, für die ebenfalls gute Behandlungsergebnisse vorliegen. Dagegen ist die Datenlage für die Hochfrequenzablation noch nicht ausreichend, um eine solche als Standardtherapie empfehlen zu können.

\section{Dr. med. Peter Stiefelhagen =}

- Quelle: Endoclub Nord, Hamburg 